\title{
COVID_SCREENET: COVID-19 Screening in Chest Radiography Images Using Deep Transfer Stacking
}

\author{
R. Elakkiya ${ }^{1} \cdot$ Pandi Vijayakumar $^{2}$ (D) Marimuthu Karuppiah $^{3}$ \\ Accepted: 2 March 2021 / Published online: 17 March 2021 \\ (C) The Author(s), under exclusive licence to Springer Science+Business Media, LLC, part of Springer Nature 2021
}

\begin{abstract}
Infectious diseases are highly contagious due to rapid transmission and very challenging to diagnose in the early stage. Artificial Intelligence and Machine Learning now become a strategic weapon in assisting infectious disease prevention, rapid-response in diagnosis, surveillance, and management. In this paper, a bifold COVID_SCREENET architecture is introduced for providing COVID-19 screening solutions using Chest Radiography (CR) images. Transfer learning using nine pre-trained ImageNet models to extract the features of Normal, Pneumonia, and COVID-19 images is adapted in the first fold and classified using baseline Convolutional Neural Network (CNN). A Modified Stacked Ensemble Learning (MSEL) is proposed in the second fold by stacking the top five pre-trained models, and then the predictions resulted. Experimentation is carried out in two folds: In first fold, open-source samples are considered and in second fold 2216 real-time samples collected from Tamilnadu Government Hospitals, India, and the screening results for COVID data is $100 \%$ accurate in both the cases. The proposed approach is also validated and blind reviewed with the help of two radiologists at Thanjavur Medical College \& Hospitals by collecting 2216 chest X-ray images between the month of April and May. Based on the reports, the measures are calculated for COVID_SCREENET and it showed $100 \%$ accuracy in performing multi-class classification.
\end{abstract}

Keywords Deep learning $\cdot$ COVID-19 $\cdot$ AI diagnostics tool $\cdot$ Diagnostic radiography $\cdot$ Machine learning $\cdot$ Medical diagnosis · X-rays

\section{Introduction}

As far as infectious diseases are considered, prevention, monitoring, rapid-response and management efforts to be particularly helpful in decelerating or filibustering outbreaks. Infectious diseases are very challenging to diagnose in the early stage, with excessive failure rate resulting in high fatality

Pandi Vijayakumar

vijibond2000@gmail.com

R. Elakkiya

elakkiyaceg@gmail.com

Marimuthu Karuppiah

marimuthume@gmail.com

1 School of Computing, SASTRA Deemed To Be University, Tamilnadu, Thanjavur, India

2 Department of Computer Science \& Engineering, University College of Engineering Tindivanam, Tindivanam, Tamilnadu, India

3 Department of Computer Science and Engineering, SRM Institute of Science and Technology, Delhi- NCR Campus, Ghaziabad, Uttar Pradesh, India and alarming rate of drug resistance. One of the most thrives of AI is often seen in life-threatening and time-sensitive conditions. In most of these kinds of critical situations, AI can help us better diagnose problems easily and quickly so that we can make decisions easily and act quickly. A novel Coronavirus outbreak was first reported in Wuhan City, China in November 2019 is a world pandemic. The virus spreads rapidly from human to human and it causes severe respiratory illness to the people irrespective of their age groups. Today the diagnosis is done using Reverse transcription polymerase chain reaction (RT-PCR) and it is a time consuming process. In most of the cases (Chu et al., 2020) the tests need to be repeated to confirm the positive corona cases because the RT-PCR detection rate is $50 \%$. Though Coronavirus or COVID-19 trends the newscast, these kinds of infectious diseases or outbreaks aren't something new for humanity and it has faced several throughout history.

In recent years, infectious diseases are more accountable with high percentile in healthcare expenditures, appreciably impacting the health of individuals and societal communities. Before COVID-19, three major outbreaks were documented: Ebola (2014-2016) reported 28,646 cases, SARS (2002- 
2004) reported with 8098 cases and MERS (2012) reported with 2494 cases. Based on the documentation of parasitic infectious diseases over 3.9 million hospital outpatientdepartment got visited in 2011 and 2014, for the same reason over 17.8 million people visited physicians in their respective offices. In 2014, many other illness were documented (Engelkirk et al., 2020) with new cases which includes Tuberculosis (9421 cases), Salmonella cases (51,455 cases), Lyme disease (33,461 cases) and meningococcal disease (433 cases).

Researchers now started to use AI to pinpoint the environmental and epidemiological issues that pave way for epidemics and to predict the infectious disease outbreaks earlier before they materialize. Radio-Imaging departments in healthcare conveniences are being charged with the amplified load created by the virus. Deep Learning solutions are having extensive variety of applications which includes Internet of Vehicles (Chen et al., 2018), distributed cloud storage management (Yan et al., 2020), mobile edge computing (Huang et al., 2020) and especially more significant in diagnosing the findings on medical imaging (Chang, 2018; Ma \& Mao, 2020) health analytics ( $\mathrm{Li}$ et al., 2020; Alsharif et al., 2020a) and drug predictions (Alsharif et al., 2020b). Henceforth, AIpowered diagnostic tool using chest X-rays were developed by various researchers to reduce the burden of RT-PCR test.

More recently researchers aimed in delivering AI-solutions using deep learning techniques to diagnose the COVID-19 patients from healthy and other pneumonia patients. AbdelBasset et al. (2020) used deep learning algorithm to optimize the image segmentation problem in chest x-rays. Following that Abdel-Basset et al. (2021a, b) introduced a disruptive technique to analyse Covid-19 infections. Wang and Wong (2020) designed and tailored a COVID_Net architecture to diagnose COVID_19 using deep CNN and reported an accuracy of $93.3 \%$. Khan et al. (2020) proposed CoroNet, a deep CNN solution to detect the COVID infectious from healthy and pneumonia patients as a multiclass classification problem. They achieved an accuracy of $89.6 \%$ and $95 \%$ for four class classification and three class classification respectively. Zhang et al. (2020) interestingly classified viral pneumonia cases with COVID-19 and without COVID-19 from typical cases using Confidence aware anomaly detection (CAAD) and reported the sensitivity of $71.70 \%$. Karim et al. (2020) proposed DeepCOVIDEplainer, an explainable deep learning architecture for diagnosing COVID-19 and achieved $96.12 \%$ of precision and $94.3 \%$ of recall values. Dhiman et al. (2021) introduced ADOPT which is an optimization based approach to diagnose the COVID-19 using chest x-rays. Similarly, AbdelBasset et al. (2021) introduced FSS-2019-nCov to perform semi-supervised segmentation of COVID-19 infection.

Due to lack of COVID-19 data availability, many researchers started extracting the features of COVID-19 and applied transfer learning to screen the COVID-19 patients.
Tian et al. (2020) used feature selection and constructed the network model to perform visual data classification. Basu and Mitra (2020) used two datasets for COVID-19 classification and designed a CNN architecture for first data to classify normal from COVID-19 and applied transfer learning for secondary data containing pneumonia and other cases and showed an accuracy of around 95\%. Farooq and Hafeez (2020) implemented the 3-step technique to fine-tune the Resnet50 architecture and named COVID_Resnet by resizing the input image into 128X28, 224X224 and 229X229 with three colour channel and achieved an accuracy of $96.23 \%$. Ucar and Korkmaz 2020 proposed COVIDiagnosis-Net using Squeezenet architecture with Bayes optimization and produced an accuracy of $98.26 \%$. Luz et al. (2020) solved a hierarchical classification problem of diagnosing pneumonia from normal in stage one and COVID-19 from pneumonia in phase 2 using EfficientNet by modifying the operators and achieved an accuracy of $93.9 \%$. Kassani et al. (2020) used pre-trained ImageNet models for feature extraction and used six machine learning models for classification and got the highest accuracy of 99\% with DenseNet121 and Bagging Tree Classifier. Apart from Covid-19 diagnosis, transfer learning and ensemble learning proved its efficiency in other medical image diagnosis (Kuko \& Pourhomayoun, 2020) and entity recognition (Peng et al., 2019).

Many researchers used available pre-trained ImageNet models and compared their performance to automate the detection of COVID-19 virus from chest radiography images. Hall et al. (2020) used pre-trained neural networks which includes Resnet50 and VGG16 for medical image classification of COVID-19 from other Pneumonia images and showed an overall accuracy of $89.2 \%$ on minimal dataset. Hemdan et al. (2020) used seven variant architectures of VGG and Google MobileNet and their approach showed an accuracy of $90 \%$ and 60\% respectively. Apostolopoulos and Mpesiana (2020) used transfer learning CNN from MobileNetV2 and VGG19 to extract the features of COVID-19, bacterial pneumonia and normal cases and their approach showed an accuracy of 96.78\%. Horry et al. (2020) used four pre-trained ImageNet models for COVID-19 diagnosis and showed that VGG19 produces good results in prediction with a precision of $83 \%$. Chowdhury et al. (2020) used eight pre-trained neural networks to perform diagnosis between normal and COVID-19 and normal, pneumonia from COVID-19 i.e. two class and three class classification problems and got an accuracy of $99.7 \%$ and $97.9 \%$ respectively. Razzak et al. (2020) used nine pre-trained ImageNet models for diagnosing COVID-19. The authors validated their data in three ways: Binary class classification, three class classification and four class classification and they got the highest accuracy of $98.75 \%$ with Resnet 101 . However, the availability of deep learning solutions to detect and diagnose the COVID-19 is still limited. Therefore, this research work aims in proposing a new deep learning solution 
COVID_SCREENET to assist radiologists in screening COVID-19 patients from normal and other pneumonia patients using chest radiography images. The contributions of the proposed COVID_SCREENET are summarized as follows:

- A generic feature representation to discriminate the COVID-19 patients from normal and other pneumonia patients. Used the predicted class labels of transfer learning as input features for stacking to perform final classification. To the best of our knowledge, this is the first study introducing the stacking with transfer learning named Modified Stacked Ensemble Learning.

- Due to lack of COVID-19 data availability, data augmentation is done to reduce generalization error, to avoid overfitting and also to reduce the bias of the model towards one class because the data distribution is not equal in all three categories.

- With extensive experiments of quantitative and qualitative analysis, the COVID_SCREENET outperforms and the detection time is also very less compared to other stateof-the-art models with satisfactory accuracy is a superior advantage in medical imaging analysis.

- Finally, an efficient end-to-end deep learning application is developed to assist radiologists in screening and diagnosing COVID-19 patients using transfer learning and stacking from normal and other viral and bacterial pneumonia patients.

The rest of the paper is organized as follows: Section 2 presents the proposed COVID_SCREENET architecture and discusses the two phases of the architectures and its components. Section 3 details the data acquisition and formation of dataset consisting COVID-19, pneumonia and normal images from heterogeneous sources. Also, discusses the experimentation carried out in the data and results of proposed COVID_SCREENET. Finally, section 4 elaborates the summary of the proposed work.

\section{Proposed COVID_SCREENET Architecture}

The proposed COVID-SCREENET architecture mainly comprises of two phases: Phase 1 of COVID_SCREENET begins with the data acquisition process which concentrates on accumulating Chest X-ray image data from multivariate data sources. Phase 1 focused on applying transfer learning using nine available CNN models in ImageNet which includes Xception net, VGG16, Resnet50, Resnet50V2, Inception_V3, Mobilenet_V2, Densenet121, Resnet101V2 and Inception_Resnet_V2net. All the models extracted the features of training and testing data comprises of normal, pneumonia and COVID-19 chest X-ray images. Phase 2 focused on performing ensemble learning on the selected five models from phase 1 transfer learning and then multi-class classification will be performed on chest X-ray images to classify the normal from abnormal categories. The proposed bifold architectural framework of COVID_SCREENET is shown in Fig. 1.

\subsection{Data Acquisition and Data Augmentation}

The phase 1 of COVID_SCREENET begins with the collection of 7725 multi-variate data from heterogeneous data sources. The Chest radiography images comprises of three categories: normal, pneumonia and COVID-19. Once the data has been collected, data pre-processing has been done with the images to rescale the shape into $224 \mathrm{X} 224$ pixels. The preprocessed images are augmented using ImageDataGenerator and also augmented with albumentations to reach a total of 6758 samples. Figure 2 denotes the sample data with and without augmentation.

\subsection{Parameter Based Homogeneous Transfer Learning: Problem Formulation}

The generated samples are fed into the transfer learning block comprising nine available CNN models with the baseline CNN to perform the multi-class classification. Let's begin with the formalized approach of transfer learning (Pan \& Yang, 2009) and visualize the building blocks of proposed baseline $\mathrm{CNN}$ with transfer learning for Chest X-ray images. Consider the domain $\mathcal{D}$ which consists of feature space $X$ where $X=\left\{x_{1}, \ldots, x_{n}\right\}$ and a marginal probability distribution $\mathrm{P}(\mathcal{X})$ such that $\mathcal{D}=\{X, \mathrm{P}(X)\}$. For a given specific domain $\mathcal{D}$, a task $\mathcal{T}=\{Y, f()$.$\} consisting of two parts: Y, a label$ space and $\mathrm{f}($.$) , a predictive function which is not an inference$ but it is learned from the training data $\left(x_{\mathrm{i}}, \mathrm{y}_{\mathrm{i}}\right)$ where $\mathrm{i} \in\{1,2,3$, $\ldots, \mathrm{N}\}$ and $\left\{\left(x_{\mathrm{i}} \in X\right),\left(\mathrm{y}_{\mathrm{i}} \in \mathrm{Y}\right)\right\}$. The task can also be rewritten in probabilistic viewpoint using the predictive function $\mathrm{f}\left(x_{\mathrm{i}}\right)=$ $\mathrm{p}\left(\mathrm{y}_{\mathrm{i}} \mid x_{\mathrm{i}}\right)$ as $\mathcal{T}=\{Y, \mathrm{P}(\mathrm{Y} \mid X)\}$.

For a given source domain $\mathcal{D}_{s}$, a task $\mathcal{T}_{s}$ and a label space $\mathrm{s}$ and a target domain $\mathcal{D} t$, a task $\mathcal{T} t$ and a label space $\mathrm{t}$, the ultimate objective of the transfer learning is to improve the predictive target function $\mathrm{ft}$.(.) in learning task $\mathcal{T} t$ based on the knowledge from $\mathcal{D} s$ and $\mathcal{T} s$. But in general, it is not always the case that $\mathcal{D}_{s}=\mathcal{D}_{t}$ and $\mathcal{T}_{s}=\mathcal{T}_{t}$. If the two tasks are different, then the label spaces may also be different i.e. $\left(Y_{s} \neq Y_{t}\right)$ or the probability distribution may vary $\left(\mathrm{P}\left(Y_{s} \mid X_{s}\right) \neq \mathrm{P}\left(Y_{t} \mid X_{t}\right)\right)$. Transfer learning would be relevant and applicable only when it meets the criterion $\left|\mathcal{D}_{t}\right| \ll\left|\mathcal{D}_{s}\right|$. In our case, transfer learning is applicable because the available data in $\mathcal{D}_{t}$ is lesser than that of $\mathcal{D}_{s}$. Here, ImageNet is considered as $\mathcal{D}_{s}$ and it got trained over 14 million images belongs to 1000 classes and the data collected from three different sources belongs to three categories are considered as $\mathcal{D}_{t}$. In this case, it is a parameter based 
Fig. 1 Bifold architectural framework of COVID SCREENET

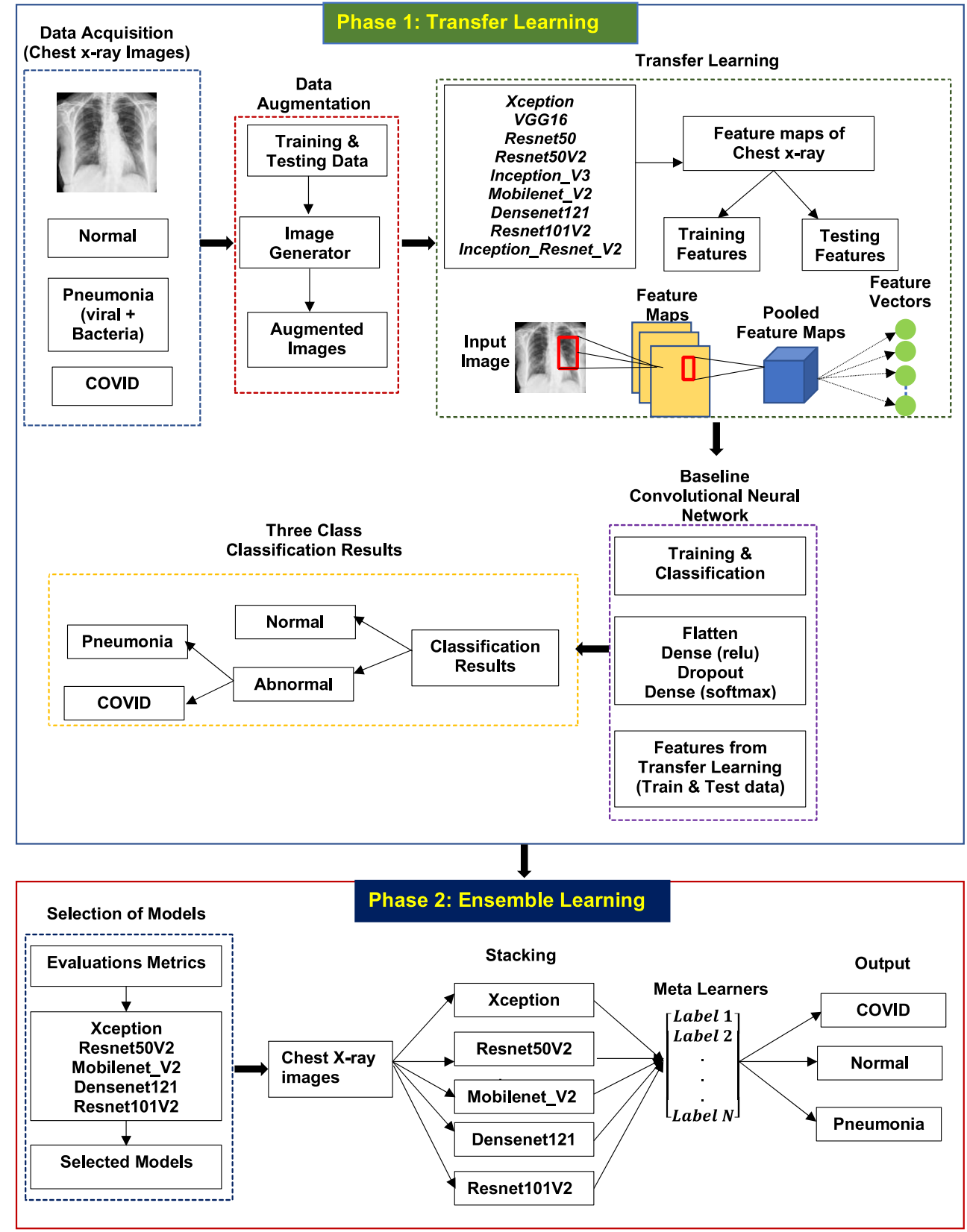

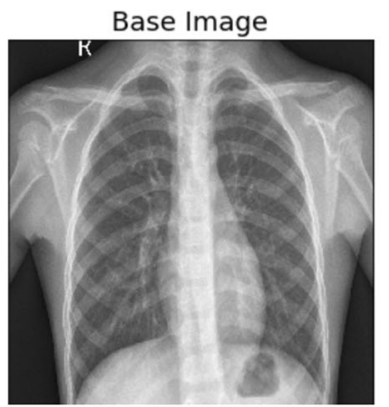
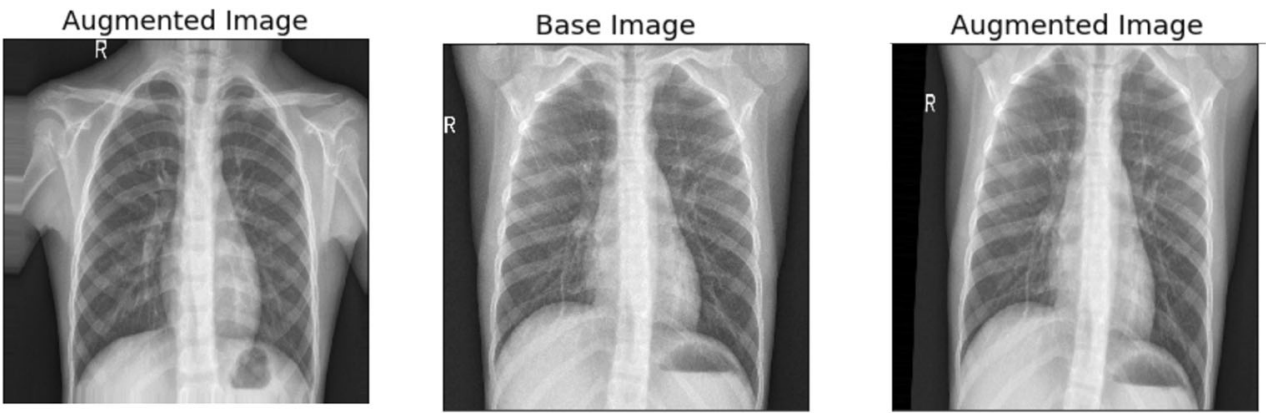

Fig. 2 Random samples of augmented images 
homogenous transfer learning and the problem $\mathcal{P}$ is defined as domain and task pair i.e. $\mathcal{P}=(\mathcal{D}, \mathcal{T})$. Here the task is learning the multi-class classification problem which constitutes the transfer learning graph as an acyclic directed graph with vertices and edges where the vertices are the problems $\mathcal{V}=\left\{\mathcal{P}_{1}, \ldots \mathcal{P}_{v}\right\}$. The aim of each transfer learning problem $\mathcal{P}_{i}=\left(\mathcal{D}_{i}, \mathcal{T}_{i}\right) \in \mathcal{V}$ is to improve the predictive function $f_{i}($.$) in$ $\mathcal{T}_{i}$.

\subsection{Baseline CNN}

The ImageNet CNN learns the low-level and high-level features from the image data from first layer to higher layer and passes on the tensors as feature vectors to baseline $\mathrm{CNN}$ which is nothing but the input to the final classifier as shown in Fig. 3. A tensor mapping of convolutional layer to feature vectors of $\mathrm{i}$-th convolutional layer $\mathcal{L}_{i}$ can be written as $\mathcal{X} \in \mathcal{R}^{h X w X d}$ to $\mathbb{C}_{i} \in \mathcal{R}^{h^{\prime} X w^{\prime} X d^{\prime}}$ therefore $\mathbb{C}_{i}=\wp_{i}\left(f_{i}\left(\mathcal{L}_{i} * \mathcal{X}+\mathcal{B}_{i}\right)\right.$ where $\mathrm{h}, \mathrm{w}, \mathrm{d}$ represents height, width and dimensions of the image, $\mathcal{L}_{i} \in \mathcal{R}^{s X s X d^{\prime}}$ is a tensor of $d^{\prime}$ filter size of $s X s$, * is the convolution operator of two-dimensions of the input, $\wp_{i}$ is the global average pooling of $2 \mathrm{D}$ inputs, $f_{i}$ is the ReLU activation function, $\mathcal{B}_{i}$ is the bias and $h^{\prime}, w^{\prime}$ are the output dimensions depends on the striding and padding of parameters.

Along with convolutional and pooling layers, the baseline CNN has fully connected dense layer in the last layer to perform classification based on the encoded feature vectors. All the nine models are trained using Adam optimizer with a learning rate of 0.001 and the losses are calculated using sparse categorical cross entropy. Once training is done, the fully connected layer will perform the classification based on the features obtained from the previous layers.

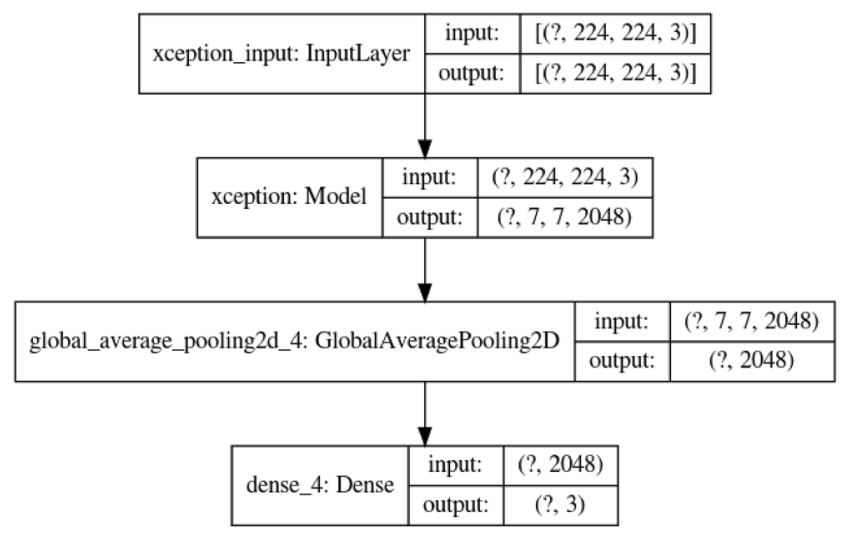

Fig. 3 Transfer learning from Xception Net to Baseline CNN

\subsection{Ensemble Learning}

The phase 2 of the proposed COVID_SCREENET framework is modified stacked ensemble learning.

Algorithm 1. MSEL.

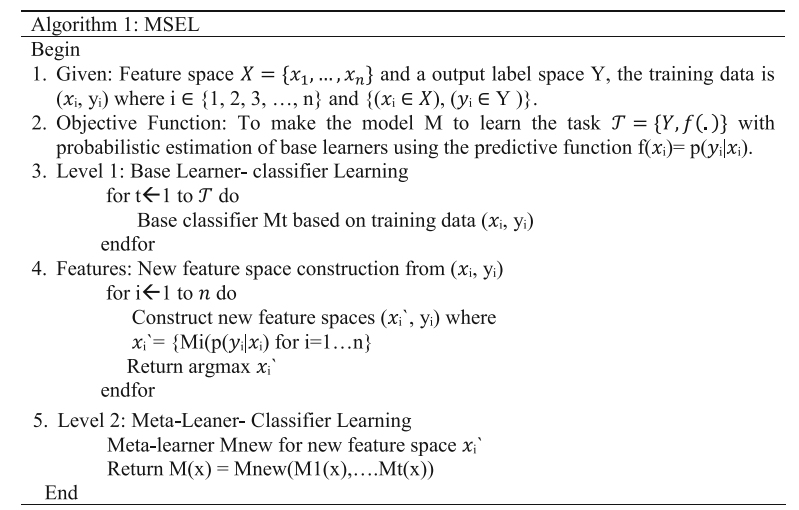

MSEL is a two-step learning process: Level 1 is training the base classifier and Level 2 is about training the meta-learners. The heterogeneous base learners are constructed using the top five models selected from phase 1 transfer learning. The selected five models are separately trained and the individual performance of these are quite encouraging. But these models are often complementary in each other when one model fails for the instance, the other one succeeds. This heterogeneity paves the way for constructing the ensemble model to get the combination all possible outputs and to improve the performance of the COVID_SCREENET framework further.

Stacked classifiers at level 1 learning started by training the original data in parallel and then it combine the results to formulate the new data for the level 2 to begin. Instead of directly taking the predicted label in the proposed framework, the probability estimation is done to predict the label based on the weightage of all learners resultant labels. The labels predicted by the base learners are the features and the actual labels are considered as class labels for the meta-learners.

In MSEL, the level 1 base learners are top five models of phase 1 transfer learning which includes Xception, Resnet50V2, Mobilenet_V2, Densenet121 and Resnet101V2. These learners will learn the data and predict the labels which will be treated as the features for the meta-learners. The meta-learner for the COVID_SCREENET is shown in Fig. 4. Based on the new data, level 2 learning of meta-learners are started to predict the output of the given data. Algorithm 1 illustrates the phase 2 stacked ensemble learning with probability estimation of base learners. 


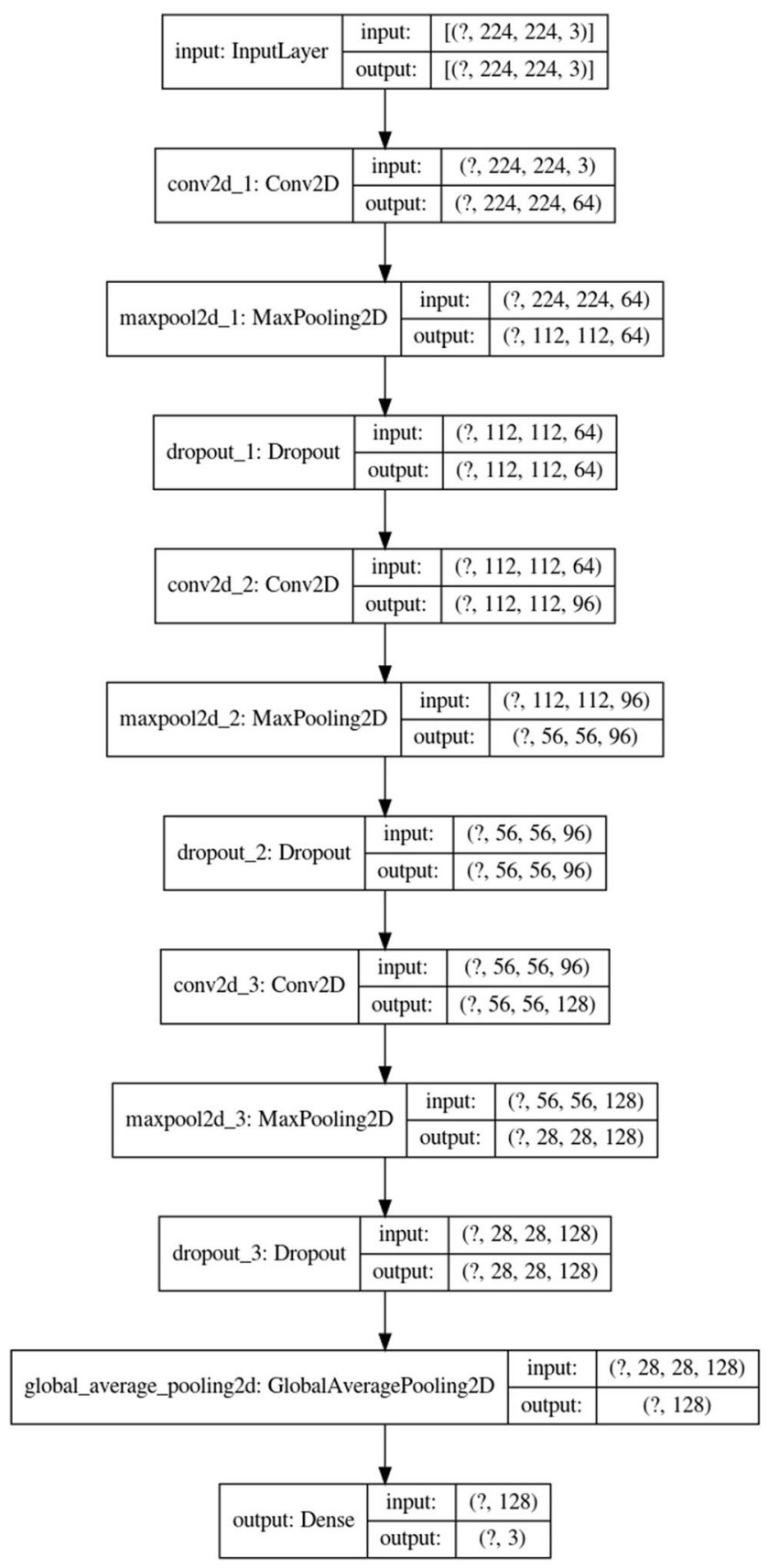

Fig. 4 Meta-Learner CNN for Modified Stacked Ensemble Learning

\section{Experimentation and Discussion}

\subsection{Datasets}

To train and test the proposed COVID-SCREENET 7725 images are collected from nine different sources. Based on $80-20$ train-test split ratio, $80 \%$ of the samples i.e. 6180 chest X-ray images are used for training and $20 \%$ of the samples i.e. 1545 images are used for testing collected. The number of data collected for each class are in the ratio of 4188 images for normal, 2928 images for Pneumonia and 609 images for COVID-19 of which 3350 images are training the normal, 2342 images are used for training the normal and 488 for training the COVID-9 classes respectively. The rest of the images in each class are used for testing the performance of the classifiers. The sample images of all the three classes are shown in Fig. 5.

The data collected from different sources with the appropriate numbers are depicted in Table 1 . The reason behind data collection from heterogeneous data sources is: the developed AI tool should be demographic supportable and it should be made available to people from all part of the world. The data distribution is not uniform with respect to number of images taken in the classes and is not quantitative enough to go with fine-tuned deep learning models. Keeping that as the primary reason, data augmentation has been done before training the samples and applied transfer learning irrespective of other deep learning concepts.

\subsection{Data Augmentation and Pre-Processing}

The pre-trained nine network models of CNN family has been trained over 14 million coloured images on ImageNet consisting of 1000 different classes. COVID_SCREENET applied transfer learning strategy to transfer the learned weights, biases and features to the baseline CNN consisting of global average pooling 2D and fully connected layers of 64 units to classify the given input data into three classes consisting of Normal, Pneumonia and COVID-19. Transfer Learning has been implemented by removing the last layer of the pretrained network and supplementing the extracted features to the baseline CNN. The input images are augmented and preprocessed by resizing and cropping to width and height of $224 X 224$ and recolouring to the colour channel of 3.

To ensure the performance of pre-processing before proceeding with complete transfer learning, a sample validation check has been experimented with the Resnet50 model. The performance of the Resnet50 model is experimented for 30 iterations with augmented images and without augmented images. The obtained results for augmentation and non-augmentation with respect to the model accuracy and loss are shown in Fig. $6 \mathrm{a}$ and $\mathrm{b}$ respectively. From the results, it is clearly seen that the model outperforms in classifying the multiclass images when it is augmented than with the images which is not augmented. Henceforth, the rest of the models are validated with augmented images and the results are discussed in the next section in detail. 


\section{NORMAL}
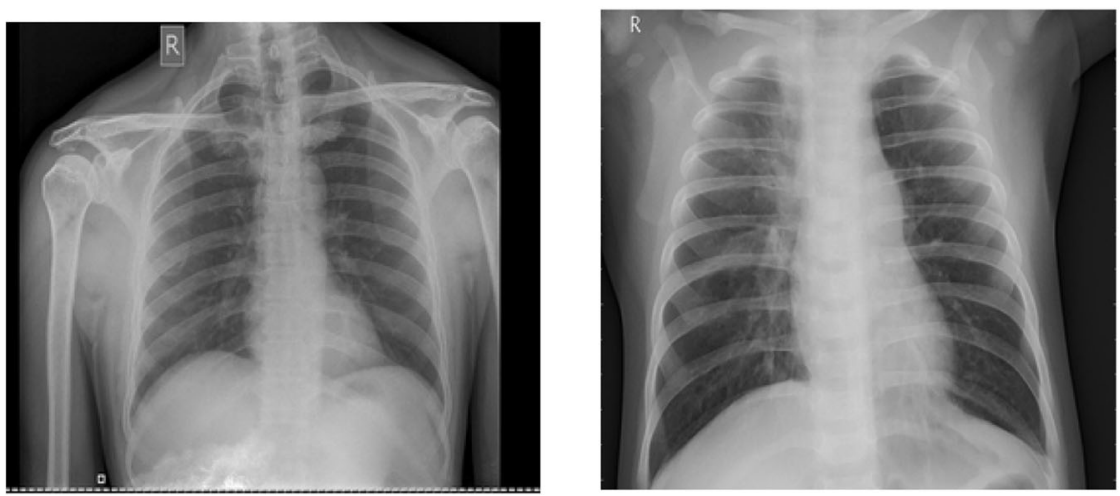

COVD

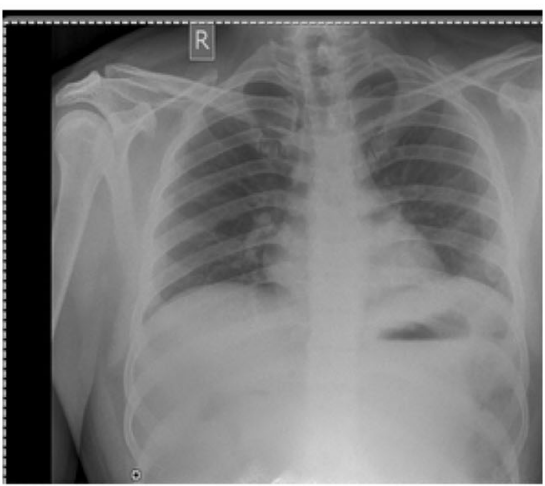

Fig. 5 Random samples of normal, pneumonia and COVID images

\subsection{Transfer Learning}

ImageNet is a pretrained neural network with more than 14 million coloured images of 1000 classes. The proposed COVID_SCREENET is not having sufficient data to train and construct a new classifier for screening COVID-19 patients, transfer learning is applied to extract the features of normal, pneumonia and COVID-19 patients using nine available ImageNet models which includes Xception net, VGG16, Resnet50, Resnet50V2, Inception V3, Mobilenet V2, Densenet121, Resnet101V2 and Inception_Resnet_V2net. Transfer learning networks are modified with a set of parameters which includes batch size of 16 , number of iterations restricted with 20 to reduce the time complexity, batches are shuffled for each iteration, learning rate of 0.001 , buffer size to 512 with the validation frequency of 5 times.

To give the desired credibility of the findings, testing has been done with the consideration of 1545 images consisting of all three class labels. Here the test images used are from different sources obtained from various countries with different device settings. The performances of the COVID_SCREENET are measured using Accuracy as in (1), Precision as in (2), Recall as in (3) and F1-Score as in (4) and the results are tabulated in Table 2.

$$
\begin{aligned}
& \text { Accuracy }=\frac{I I+N N}{I I+N N+I N+N I} \\
& \text { Precision }=\frac{I I}{I I+I N} \\
& \text { Recall }=\frac{N N}{N N+N I} \\
& F 1-\text { Score }=2 X \frac{\text { Precision } * \text { Recall }}{\text { Precision }+ \text { Recall }} \mathrm{v}
\end{aligned}
$$

Based on the results depicted in Fig. 7 and Table 2, it is

\begin{tabular}{|c|c|c|c|c|c|}
\hline S. No & Source & Number of Xray images & Normal & Pneumonia & $\begin{array}{l}\text { COVID- } \\
19\end{array}$ \\
\hline 1 & $\begin{array}{l}\text { https://www.kaggle.com/tawsifurrahman/covid19-radiography-database } \\
\text { (Chowdhury et al., 2020) }\end{array}$ & 2905 & 1341 & 1345 & 219 \\
\hline 2 & https://www.kaggle.com/nih-chest-xrays/data (Wang et al. 2017) & 764 & 764 & - & - \\
\hline 3 & $\begin{array}{l}\text { http://www.chestx-ray.com/index.php/education/normal-cxr-module- } \\
\text { train-your-eye\#!10 }\end{array}$ & 500 & 500 & - & - \\
\hline 4 & https://data.mendeley.com/datasets/rscbjbr9sj/2 (Kermany et al. 2018) & 3166 & 1583 & 1583 & - \\
\hline 5 & https://www.sirm.org/category/senza-categoria/covid-19/ & 88 & - & - & 88 \\
\hline \multirow[t]{2}{*}{6} & $\begin{array}{l}\text { https://radiopaedia.org/ } \\
\text { https://www.eurorad.org/ }\end{array}$ & 220 & - & - & 220 \\
\hline & $\begin{array}{l}\text { https://www.ams.edu.sg/colleges/radiologists/covid-19-resource-site-for- } \\
\text { radiology-imaging (Cohen et al., 2020) }\end{array}$ & & & & \\
\hline 7 & Data from Three Govt. Hospitals (Thanjavur, Theni, Tirunelveli) at Tamilnadu & 82 & - & - & 82 \\
\hline Total & 7725 & 4188 & 2928 & 609 & \\
\hline
\end{tabular}
inferred that based on the accuracy, the top five models are Xception, Resnet50, Mobilenet_V2, Densenet121 and

Table 1 Data acquisition from heterogeneous sources 
a

a Accuracy, 7 Conv Layers W/ \& W/out Image Augmentation

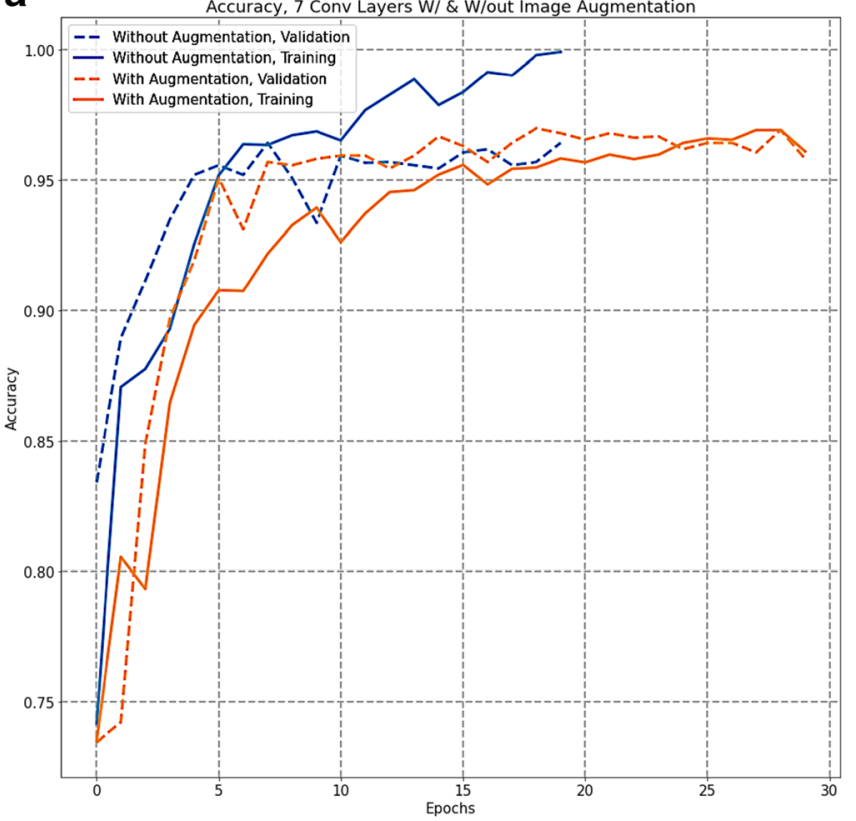

b

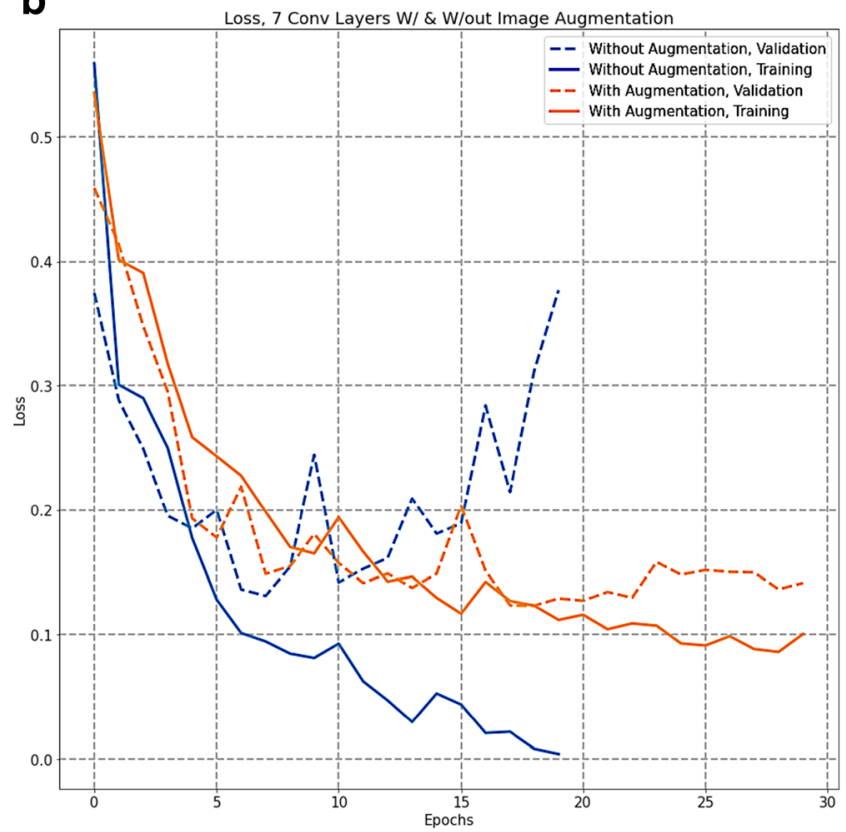

Fig. 6 Resnet50 with and without augmentation a Accuracy and b Loss

Table 2 Performance measures of transfer learning

\begin{tabular}{|c|c|c|c|c|c|}
\hline Model & Class & Precision & Recall & $\begin{array}{l}\text { F1- } \\
\text { Score }\end{array}$ & Accuracy \\
\hline \multirow[t]{3}{*}{ Xception } & COVID-19 & 1.00000 & 0.97917 & 0.98947 & \multirow[t]{3}{*}{0.99656} \\
\hline & NORMAL & 0.99643 & 1.00000 & 0.99821 & \\
\hline & Pneumonia & 0.99606 & 0.99606 & 0.99606 & \\
\hline \multirow[t]{3}{*}{ VGG16 } & COVID-19 & 0.92000 & 0.85185 & 0.88462 & \multirow[t]{3}{*}{0.93804} \\
\hline & NORMAL & 0.95019 & 0.93939 & 0.94476 & \\
\hline & Pneumonia & 0.92963 & 0.95437 & 0.94184 & \\
\hline \multirow[t]{3}{*}{ Resnet50 } & COVID-19 & 0.94872 & 0.97368 & 0.96104 & \multirow[t]{3}{*}{0.97074} \\
\hline & NORMAL & 0.98473 & 0.95911 & 0.97175 & \\
\hline & Pneumonia & 0.96071 & 0.98175 & 0.97112 & \\
\hline \multirow[t]{3}{*}{ Resnet50V2 } & COVID-19 & 1.00000 & 1.00000 & 1.00000 & \multirow[t]{3}{*}{0.96902} \\
\hline & NORMAL & 0.97112 & 0.96416 & 0.96763 & \\
\hline & Pneumonia & 0.96094 & 0.96850 & 0.96471 & \\
\hline \multirow[t]{3}{*}{ Inception_V3 } & COVID-19 & 0.97826 & 1.00000 & 0.98901 & \multirow[t]{3}{*}{0.96730} \\
\hline & NORMAL & 0.98864 & 0.94565 & 0.96667 & \\
\hline & Pneumonia & 0.94465 & 0.98462 & 0.96422 & \\
\hline \multirow[t]{3}{*}{ Mobilenet_V2 } & COVID-19 & 0.97872 & 0.95833 & 0.96842 & \multirow[t]{3}{*}{0.98107} \\
\hline & NORMAL & 0.98566 & 0.98566 & 0.98566 & \\
\hline & Pneumonia & 0.97647 & 0.98031 & 0.97839 & \\
\hline \multirow[t]{3}{*}{ Densenet121 } & COVID-19 & 1.00000 & 0.97778 & 0.98876 & \multirow[t]{3}{*}{0.97762} \\
\hline & NORMAL & 0.96479 & 0.99275 & 0.97857 & \\
\hline & Pneumonia & 0.98814 & 0.96154 & 0.97466 & \\
\hline \multirow[t]{3}{*}{ Resnet101V2 } & COVID-19 & 0.96000 & 1.00000 & 0.97959 & \multirow[t]{3}{*}{0.97074} \\
\hline & NORMAL & 0.98519 & 0.95341 & 0.96903 & \\
\hline & Pneumonia & 0.95785 & 0.98425 & 0.97087 & \\
\hline \multirow{3}{*}{$\begin{array}{l}\text { Inception_Resnet_ } \\
\text { V2 }\end{array}$} & COVID-19 & 1.00000 & 0.88889 & 0.94118 & \multirow[t]{3}{*}{0.93287} \\
\hline & NORMAL & 0.99180 & 0.87681 & 0.93077 & \\
\hline & Pneumonia & 0.87542 & 1.00000 & 0.93357 & \\
\hline
\end{tabular}



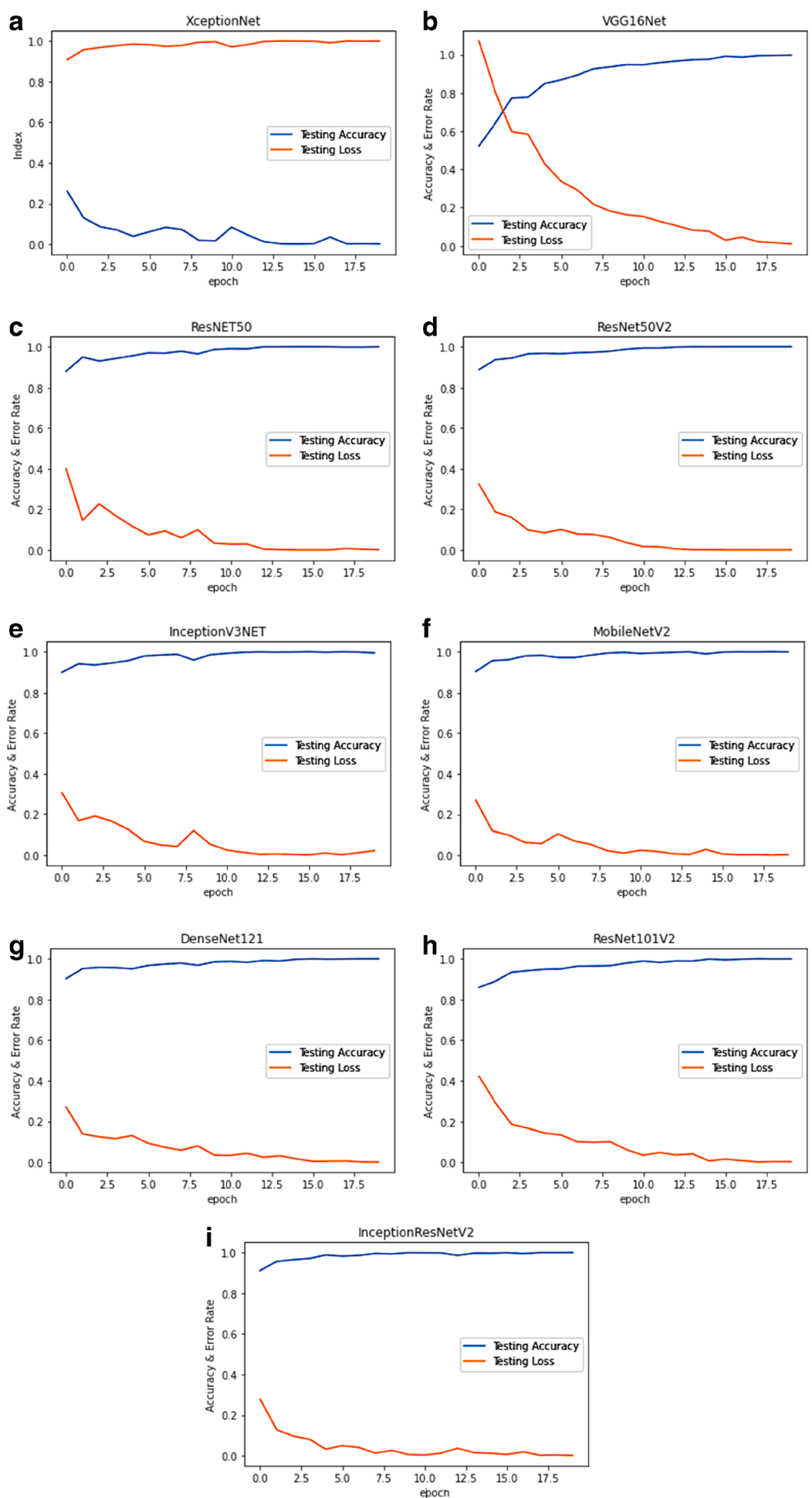

Fig. 7 Testing loss and accuracy of transfer learning a Xception net, bVGG16, c Resnet50, d Resnet50V2, e Inception_V3, f Mobilenet_V2, g Densenet121, h Resnet101V2 and i Inception_Resnet_V2net 
Resnet101V2 with an accuracy of 99.7\%, 96.9\%, 98.1\%, $97.8 \%$ and $97 \%$ respectively. But in medical image analysis it is not always the fact of using the model with best accuracy, it is matter of fact to consider the model with best sensitivity and specificity rate so as to avoid the questioning of false positive and false negative predictions. With this interpretation, the precision and recall rate of Resnet50V2 is $100 \%$ for COVID-19 class whereas for Resnet50 it is $95 \%$ and $97 \%$ respectively. The comparison clearly shows the Resnet50V2 predicts the samples with $100 \%$ sensitivity and specificity and the accuracy also closer to Resnet50 with a variation of $0.172 \%$. Therefore the top five models based on transfer learning performance are chosen for further classification using Ensemble Learning includes Xception, Resnet50V2, Mobilenet_V2, Densenet121 and Resnet101V2 and the corresponding confusion matrix is shown in Fig. 8.

\subsection{Modified Stacked Ensemble Learning}

From phase 1 transfer learning, the best five models with top performance such as Xception, Resnet50V2, Mobilenet_V2, Densenet121 and Resnet101V2 are selected based on various metrics. These models are the base learners in MSEL level 1 learning of COVID_SCREENET. The input images of all the three classes are pre-processed with height and width of 224 pixels and three colour channels have been chosen. Level 1 learning follows the same experimental set up as like transfer learning. The class labels are predicted individually by base learners and served as the input to meta-learner $\mathrm{CNN}$ to make final predictions.

The results of base learners and the resultant class predicted by meta-learner of MSEL is shown in Fig. 9 and the performance of the MSEL classifier with the confusion matrix is shown in Fig. 10. From the obtained results, it is clearly shown that the proposed COVID_SCREENET produced an accuracy of $100 \%$ in classifying the multiclass classification of COVID-19, Normal and Pneumonia classes.

Though as a single classifier fails to predict the correct label, the proposed MSEL using probability estimation finds the exact class labels of the input images and produced $100 \%$ accurate results in real-time samples collected from heterogeneous sources.

\subsection{COVID_SCREENET Diagnostics Tool}

The proposed COVID_SCREENET framework is developed and delivered as an AI diagnostics tool to perform the early diagnosis of COVID-19 screening before performing RT-PCR test. Generally, RT-PCR test costs are higher when compared to X-Ray costs and moreover the time taken by RT-PCR is restricted to minimum of $7 \mathrm{~h}$ (https://indianexpress.com/article/explained/coronaviruscovid-19-testing-procedures-in-india-6479312/, n.d.).
When the number of test samples are more, the time taken to get the results will also get increased. To avoid such delay in prediction, the proposed COVID_SCREENET is developed as a diagnostics tool to make the predictions lesser than the second. This tool is initially validated with the real time samples collected (https://www.sirm.org/ category/senza-categoria/covid-19/). Next, the validation was done on the samples collected from three different districts of Tamilnadu, India which includes Thanjavur medical college \& Hospitals (61 COVID-19 samples), Theni Government Medical College (37 COVID-19 Samples), and Tirunelveli Medical College (20 COVID19 Samples). The tool differentiated accurately the COVID-19 images from normal and pneumonia images.

On May $18 \mathrm{th}, 2020$ the tool (https:// timesofindia.indiatimes.com/city/chennai/tamil-nadu-university-develops-ai-based-software-for-covid-19-preliminaryscreening/articleshow/76088643.cms, n.d.; https:// www.newindianexpress.com/states/tamil-nadu/2020/may/20/ sastras-ai-based-screening-filter-to-validate-need-for-rt-pcrtest-2145687.html, n.d.) was deployed at Thanjavur medical college and the training session on usage of the tool was given to the Doctors. Using this tool, the doctors can upload a folder of images or a single image and see the prediction results. For analysing a single image, the tool is taking $0.23 \mathrm{~s}$. Henceforth the tool can diagnose 100 patients in less than $25 \mathrm{~s}$ and deliver the result in a spreadsheet with patient ID followed by the categories as Normal/Abnormal and if it is abnormal then further classification of COVID/Pneumonia. The proposed COVID-SCREENET tool is shown in Fig. 11.

\section{Conclusion}

COVID-19 is a world pandemic infectious disease because of its rapid transmission and the diagnosis of such infectious disease is a key role to control community transmission. In this paper, deep learning-based COVIDSCREENET architecture is introduced to diagnose the COVID-19 infection using Chest Radiography images. The proposed approach followed a bifold-architecture to discriminate the radiological features of chest X-rays using transfer learning in phase one and to classify the COVID-19 other pneumonia infections and normal people using MSEL. A multi-variate dataset has been created by collecting data from multiple sources and extensive experiments have been conducted to emphasize the performance of the proposed COVID_SCREENET. The experimental results of the proposed approach showed $99 \%$ and $100 \%$ accuracy in detecting and performing multi-class classification using transfer learning and MSEL respectively. 

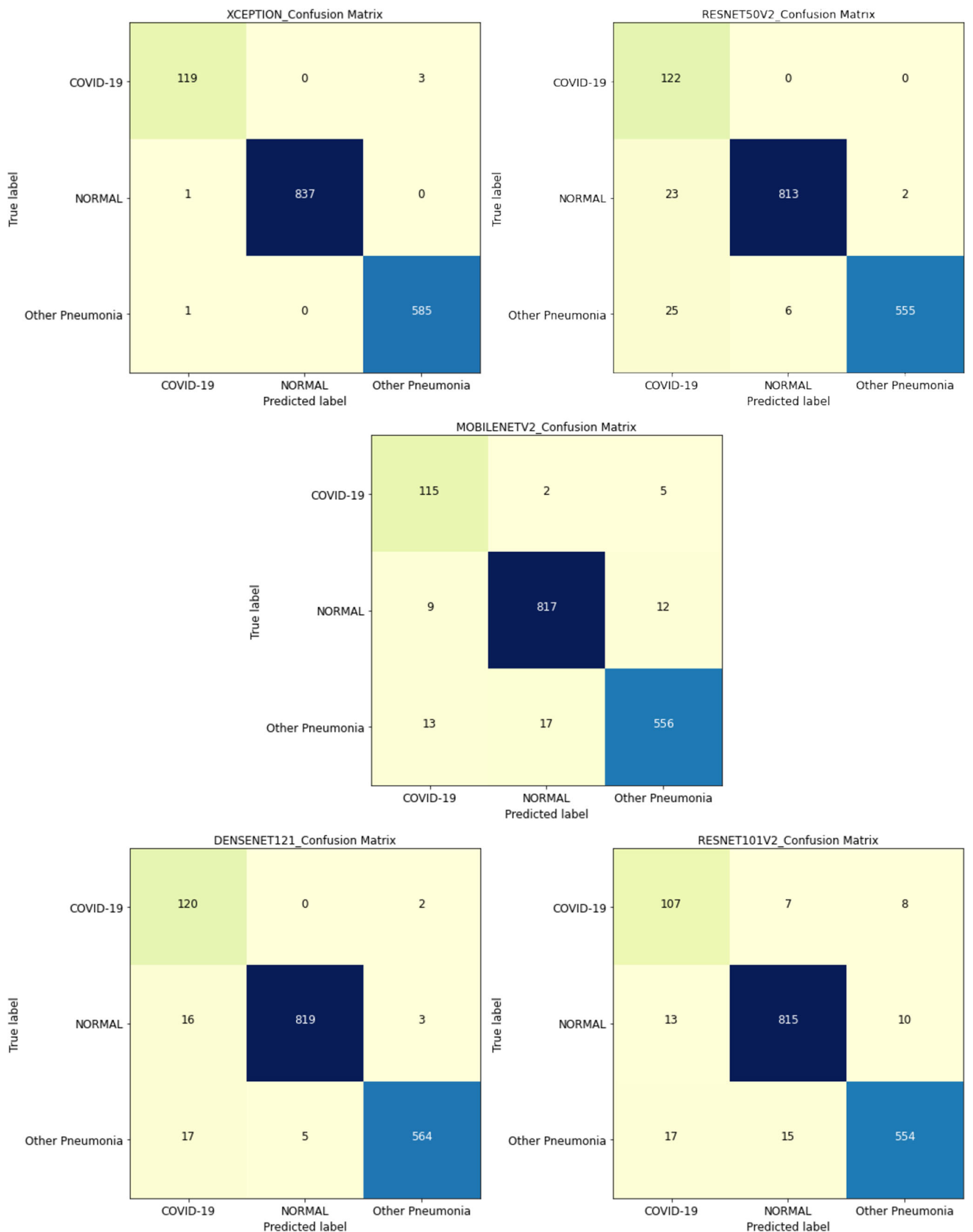

Fig. 8 Phase 1-COVID_SCREENET transfer learning confusion matrix 


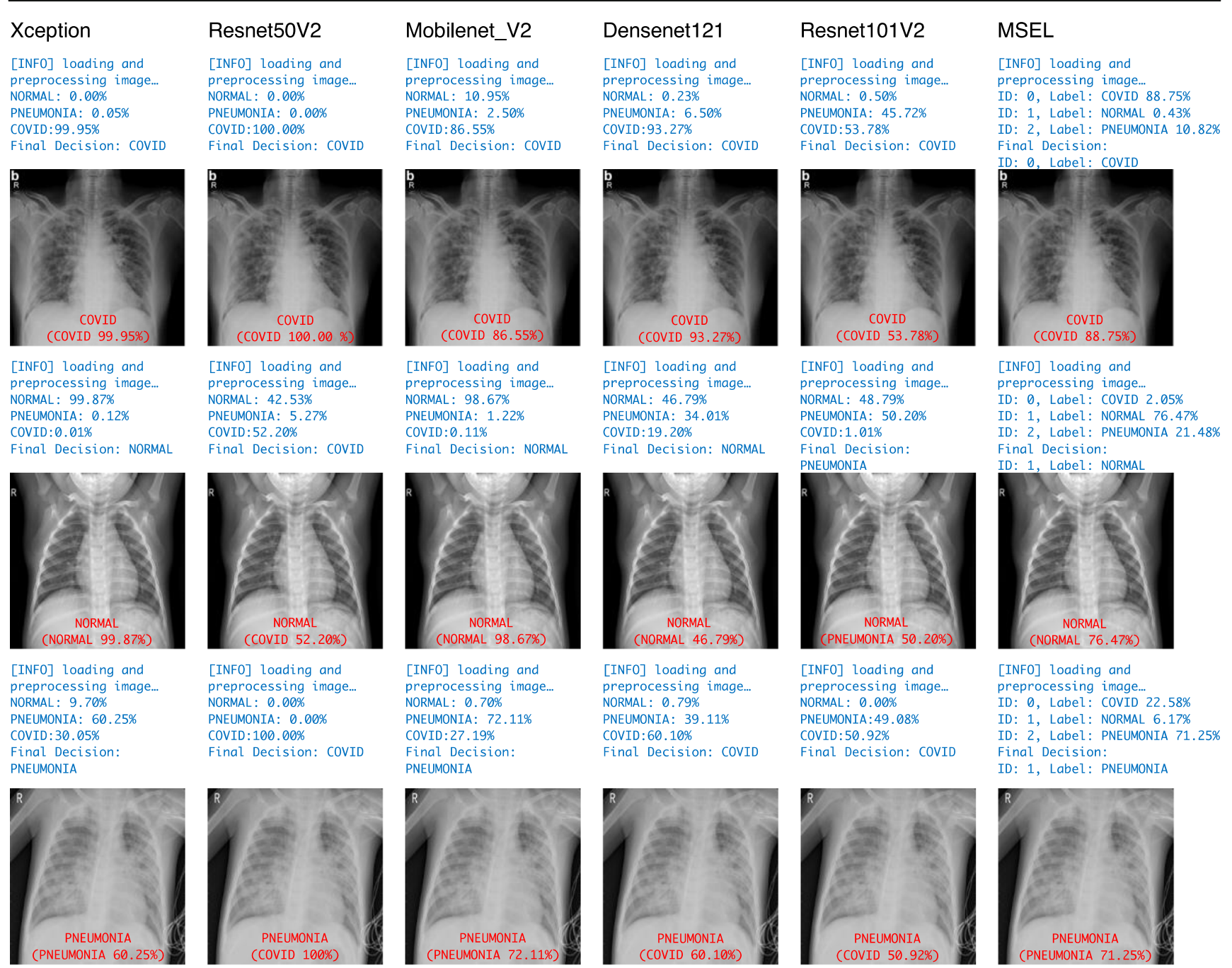

Fig. 9 Multiclass Classification using Modified Stacked Ensemble Learning

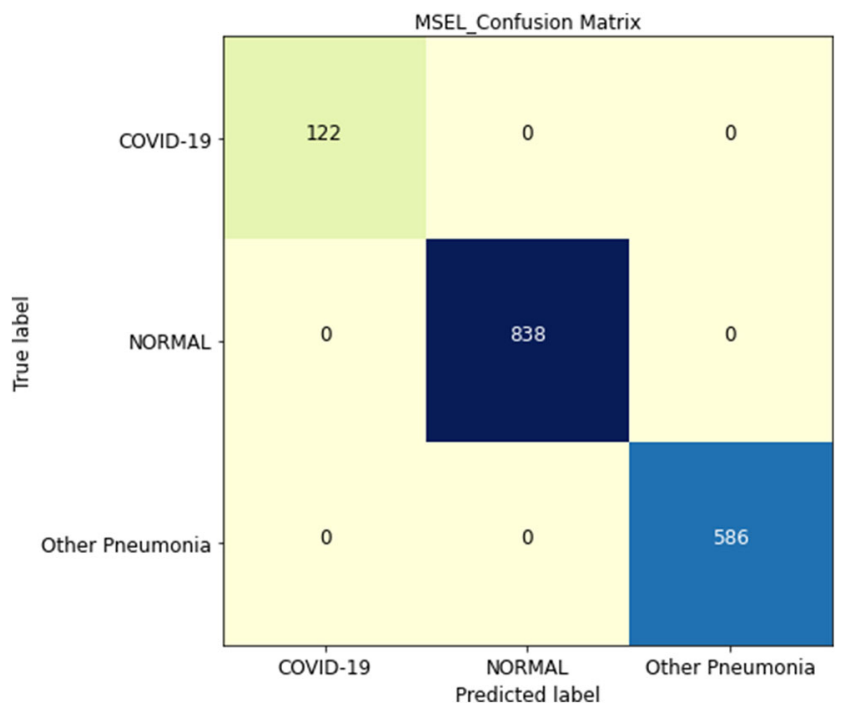

Fig. 10 Phase 2-COVID_SCREENET MSEL confusion matrix
The proposed COVID_SCREENET has been successfully developed as a sophisticated end-to-end AI-based diagnostics tool to detect and diagnose the COVID-19 in less than one second. The tool has been deployed in Government Medical Colleges in Tamilnadu, India and tested with real-time samples collected from the patients visited the hospitals. The tool outperformed in diagnosing and screening the COVID-19 patients and the results obtained from the tool is highly correlating with the predictions of the Radiologists. The tool is diagnosing the patient's X-ray images faster than RT-PCR, it can be used to control the transmission of COVID-19 thereby test demand using RT-PCR is avoidable. Henceforth it is proved that this tool will help in accurate preliminary screening and therefore, it can be used as a filter before recommending the RT-PCR test. 


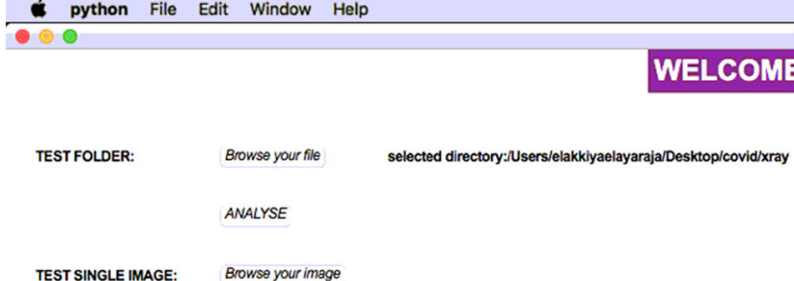

COVID
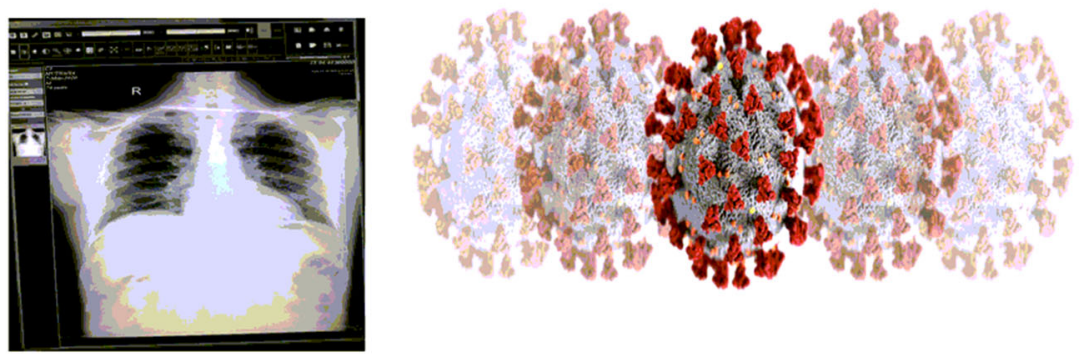

COPYRIGHTS RESERVED @ E-SIKSha, FIRST TBI, SASTRA DEEMED TO BE UNIVERSITY, THANJAVUR

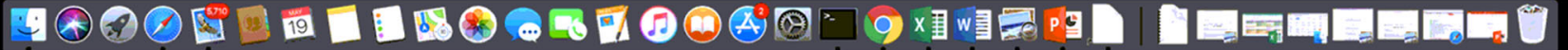

Fig. 11 COVID_SCREENET diagnostics solutions

\section{References}

Abdel-Basset, M., Chang, V., \& Mohamed, R. (2020). HSMA WOA: A hybrid novel slime mould algorithm with whale optimization algorithm for tackling the image segmentation problem of chest X-ray images. Applied Soft Computing, 95, 106642.

Abdel-Basset, M., Chang, V., \& Nabeeh, N. A. (2021a). An intelligent framework using disruptive technologies for COVID-19 analysis. Technological Forecasting and Social Change, 163, 120431.

Abdel-Basset, M., Chang, V., Hawash, H., Chakrabortty, R. K., \& Ryan, M. (2021b). FSS-2019-nCov: A deep learning architecture for semisupervised few-shot segmentation of COVID-19 infection. Knowledge-Based Systems, 212, 106647.

Alsharif, M. H., Alsharif, Y. H., Albreem, M. A., Jahid, A., Solyman, A. A. A., Yahya, K., Alomari, O. A., \& Hossain, M. S. (2020a). Application of machine intelligence technology in the detection of vaccines and medicines for SARS-CoV-2. European Review for Medical and Pharmacological Sciences, 24(22), 11977-11981.

Alsharif, M. H., Alsharif, Y. H., Yahya, K., Alomari, O. A., Albreem, M. A., \& Jahid, A. (2020b). Deep learning applications to combat the dissemination of COVID-19 disease: A review. European Review for Medical and Pharmacological Sciences, 24(21), 11455-11460.

Apostolopoulos, I. D., \& Mpesiana, T. A. (2020). Covid-19: Automatic detection from $\mathrm{x}$-ray images utilizing transfer learning with convolutional neural networks. Physical and Engineering Sciences in Medicine, 1. Available: https://www.ncbi.nlm.nih.gov/pmc/ articles/PMC7118364/
Basu, S., \& Mitra, S. (2020). Deep learning for screening COVID-19 using chest X-ray images. arXiv preprint arXiv:2004.10507. Available: https://arxiv.org/pdf/2004.10507

Chang, V. (2018). Computational intelligence for medical imaging simulations. Journal of Medical Systems, 42(1), 10.

Chen, C., Xiang, H., Qiu, T., Wang, C., Zhou, Y., \& Chang, V. (2018). A rear-end collision prediction scheme based on deep learning in the internet of vehicles. Journal of Parallel and Distributed Computing, 117, 192-204.

Chowdhury, M. E., Rahman, T., Khandakar, A., Mazhar, R., Kadir, M. A., Mahbub, Z. B., ... \& Reaz, M. B. I. (2020). Can AI help in screening viral and COVID-19 pneumonia? arXiv preprint arXiv: 2003.13145. Available: https://arxiv.org/pdf/2003.13145

Chu, D. K., Pan, Y., Cheng, S. M., Hui, K. P., Krishnan, P., Liu, Y., et al. (2020). Molecular diagnosis of a novel coronavirus (2019-nCoV) causing an outbreak of pneumonia. Clinical Chemistry, 66(4), 549555 Available: https://academic.oup.com/clinchem/article/66/4/ 549/5719336.

Cohen, J. P., Morrison, P., Dao, L., Roth, K., Duong, T. Q., \& Ghassemi, M. (2020). COVID-19 image data collection: Prospective predictions are the future. arXiv preprint arXiv:2006.11988. Available: https://arxiv.org/pdf/2006.11988

Dhiman, G., Chang, V., Kant Singh, K., \& Shankar, A. (2021). ADOPT: Automatic deep learning and optimization-based approach for detection of novel coronavirus COVID-19 disease using X-ray images. Journal of biomolecular structure and dynamics, 1-13. 
Engelkirk, P. G., Duben-Engelkirk, J., \& Fader, R. C. (2020). Burton's microbiology for the health sciences. Burlington: Jones \& Bartlett Publishers.

Farooq, M., \& Hafeez, A. (2020). Covid-resnet: A deep learning framework for screening of covid19 from radiographs. arXiv preprint arXiv:2003.14395. Available: https://arxiv.org/pdf/2003.14395

Hall, L. O., Paul, R., Goldgof, D. B., \& Goldgof, G. M. (2020). Finding covid-19 from chest $\mathrm{x}$-rays using deep learning on a small dataset. arXiv preprint arXiv:2004.02060. Available: https://arxiv.org/pdf/ 2004.02060

Hemdan, E. E. D., Shouman, M. A., \& Karar, M. E. (2020). Covidx-net: A framework of deep learning classifiers to diagnose covid-19 in $\mathrm{x}$ ray images. arXiv preprint arXiv:2003.11055. Available: https:// arxiv.org/pdf/2003.11055

Horry, M. J., Paul, M., Ulhaq, A., Pradhan, B., Saha, M., \& Shukla, N. (2020). X-ray image based COVID-19 detection using pre-trained deep learning models. Available: https://engrxiv.org/wx89s/ download?format=pdf

https://indianexpress.com/article/explained/coronavirus-covid-19testing-procedures-in-india-6479312/

https:/timesofindia.indiatimes.com/city/chennai/tamil-nadu-universitydevelops-ai-based-software-for-covid-19-preliminary-screening/ articleshow/76088643.cms

https://www.newindianexpress.com/states/tamil-nadu/2020/may/20/ sastras-ai-based-screening-filter-to-validate-need-for-rt-pcr-test2145687.html.

https://www.sirm.org/category/senza-categoria/covid-19/

Huang, B., Li, Z., Xu, Y., Pan, L., Wang, S., Hu, H., \& Chang, V. (2020). Deep reinforcement learning for performance-aware adaptive resource allocation in mobile edge computing. Wireless Communications and Mobile Computing, 2020.

Karim, M., Döhmen, T., Rebholz-Schuhmann, D., Decker, S., Cochez, M., \& Beyan, O. (2020). Deepcovidexplainer: Explainable covid-19 predictions based on chest $\mathrm{X}$-ray images. arXiv preprint arXiv: 2004.04582. Available: https://arxiv.org/pdf/2004.04582

Kassani, S. H., Kassasni, P. H., Wesolowski, M. J., Schneider, K. A., \& Deters, R. (2020). Automatic detection of coronavirus disease (COVID-19) in X-ray and CT images: A machine learning-based approach. arXiv preprint arXiv:2004.10641. Available: https:// arxiv.org/pdf/2004.10641

Kermany, D. S., Goldbaum, M., Cai, W., Valentim, C. C., Liang, H., Baxter, S. L., et al. (2018). Identifying medical diagnoses and treatable diseases by image-based deep learning. Cell, 172(5), 11221131 Available: https:/www.sciencedirect.com/science/article/pii/ S0092867418301545.

Khan, A. I., Shah, J. L., \& Bhat, M. M. (2020). Coronet: A deep neural network for detection and diagnosis of COVID-19 from chest x-ray images. Computer Methods and Programs in Biomedicine, 105581. Available: https://www.sciencedirect.com/science/article/pii/ S0169260720314140

Kuko, M., \& Pourhomayoun, M. (2020). Single and clustered cervical cell classification with ensemble and deep learning methods. Information Systems Frontiers, 22(5), 1039-1051.

Li, X., Wang, Y., Zhang, B., \& Ma, J. (2020). PSDRNN: An efficient and effective HAR scheme based on feature extraction and deep learning. IEEE Transactions on Industrial Informatics.

Luz, E., Silva, P. L., Silva, R., \& Moreira, G. (2020). Towards an efficient deep learning model for covid-19 patterns detection in X-ray images. arXiv preprint arXiv:2004.05717.Available: https://arxiv.org/pdf/ 2004.05717

Ma, M., \& Mao, Z. (2020). Deep convolution-based LSTM network for remaining useful life prediction. IEEE Transactions on Industrial Informatics.

Pan, S. J., \& Yang, Q. (2009). A survey on transfer learning. IEEE Transactions on Knowledge and Data Engineering, 22(10), 1345-
1359 Available: https://ieeexplore.ieee.org/iel5/69/4358933/ 05288526.pdf.

Peng, D., Wang, Y., Liu, C., \& Chen, Z. (2019). TL-NER: A transfer learning model for Chinese named entity recognition. Information Systems Frontiers, 1-14.

Razzak, I., Naz, S., Rehman, A., Khan, A., \& Zaib, A. (2020). Improving coronavirus (COVID-19) diagnosis using deep transfer learning. medRxiv. Available: https://www.medrxiv.org/content/medrxiv/ early/2020/04/17/2020.04.11.20054643.full.pdf

Tian, H., Chen, S. C., \& Shyu, M. L. (2020). Evolutionary programming based deep learning feature selection and network construction for visual data classification. Information Systems Frontiers, 22(5), 1053-1066.

Ucar, F., \& Korkmaz, D. (2020). COVIDiagnosis-net: Deep BayesSqueezeNet based diagnostic of the coronavirus disease 2019 (COVID-19) from X-ray images. Medical Hypotheses, 109761. Available: https://www.sciencedirect.com/science/article/pii/ S0306987720307702

Wang, L., \& Wong, A. (2020). COVID-net: A tailored deep convolutional neural network Design for detection of COVID-19 cases from chest X-ray images. arXiv preprint arXiv:2003.09871. Available: https://arxiv.org/pdf/2003.09871

Wang, X., Peng, Y., Lu, L., Lu, Z., Bagheri, M., \& Summers, R. M. (2017). Chestx-ray8: Hospital-scale chest $\mathrm{X}$-ray database and benchmarks on weakly-supervised classification and localization of common thorax diseases. In Proceedings of the IEEE conference on computer vision and pattern recognition (pp. 2097-2106). Available: http://openaccess.thecvf.com/content_cvpr_2017/ papers/Wang_ChestX-ray8_Hospital-Scale_Chest_CVPR_2017_ paper.pdf

Yan, S., He, L., Seo, J., \& Lin, M. (2020). Concurrent healthcare data processing and storage framework using deep-learning in distributed cloud computing environment. IEEE Transactions on Industrial Informatics.

Zhang, J., Xie, Y., Li, Y., Shen, C., \& Xia, Y. (2020). Covid-19 screening on chest $\mathrm{X}$-ray images using deep learning based anomaly detection. arXiv preprint arXiv:2003.12338. Available: https://arxiv.org/pdf/ 2003.12338

Publisher's Note Springer Nature remains neutral with regard to jurisdictional claims in published maps and institutional affiliations.

Elakkiya R is working as an Assistant Professor in Department of Computer Science \& Engineering, School of Computing, SASTRA University, Thanjavur. She received her Doctor of Philosophy from Anna University, Chennai in 2018. She got three patents and She has published more than 20 research articles in leading journals, conference proceedings and book including IEEE, Elsevier and Springer. Currently, she is an editor of Information Engineering and Applied Computing journal and also, she is a Life time member of International Association of Engineers

Pandi Vijayakumar received the B.E. degree in computer science and engineering from Madurai Kamaraj University, Madurai, India, in 2002, the M.E. degree in computer science and engineering from the Karunya Institute of Technology, Coimbatore, India, in 2005, and the Ph.D. degree in computer science and engineering from Anna University, Chennai, India, in 2013. He is the former Dean and currently an Assistant Professor with the Department of Computer Science and Engineering, University College of Engineering Tindivanam, Melpakkam, India, which is a constituent college of Anna University Chennai, Chennai, India. He has supervised four Ph.D. candidates successfully. He has also authored or coauthored many quality papers in various IEEE transactions/ journals, ACM transaction, Elsevier, IET, and Springer journals. His 
current research interests include key management in network security, VANET security, and multicasting in computer networks.

Marimuthu Karuppiah received his B.E. degree in Computer Science and Engineering from Madurai Kamaraj University, Madurai, India in 2003, M.E. degree in Computer Science and Engineering from Anna University, Chennai, India in 2005, Ph.D. degree in Computer Science and Engineering from VIT University, Vellore, India in 2015. Presently, he is a Professor in Department of Computing Science and Engineering,
SRM University, Delhi-NCR, India. Before joining SRMIST, he was with the VIT University, Vellore, India as an Associate Professor. He has published more than 30 research papers in SCI indexed journals. Also, he has published more than 40 research papers in SCOPUS indexed journals and international conferences. He is a life member of Cryptology Research Society of India (CRSI) and Computer Society of India (CSI), senior member of IEEE and member of ACM. His main research interests include cryptography and wireless network security, in particular, authentication and encryption schemes. 